Article

\title{
Comparison of Phytochemicals, Antioxidant, and In Vitro Anti-Alzheimer Properties of Twenty-Seven Morus spp. Cultivated in Thailand
}

\author{
Piya Temviriyanukul ${ }^{1}{ }^{\circledR}$, Varittha Sritalahareuthai ${ }^{1}$, Kriskamol Na Jom $^{2}$, Butsara Jongruaysup $^{3}$, \\ Somying Tabtimsri ${ }^{4}$, Kanchana Pruesapan ${ }^{5}$, Sirinapa Thangsiri ${ }^{1}$, Woorawee Inthachat ${ }^{1}$, \\ Dalad Siriwan ${ }^{6}$, Somsri Charoenkiatkul ${ }^{1}$ and Uthaiwan Suttisansanee ${ }^{1, *(D)}$ \\ 1 Institute of Nutrition, Mahidol University, Salaya, Phuttamonthon, Nakhon Pathom 73170, Thailand; \\ piya.tem@mahidol.ac.th (P.T.); varittha.sri@hotmail.com (V.S.); poo.sweet@hotmail.com (S.T.); \\ woorawee.int@mahidol.ac.th (W.I.); somsri.chr@mahidol.ac.th (S.C.) \\ 2 Department of Food Science and Technology, Faculty of Agro-Industry, Kasetsart University, Chatuchak, \\ Bangkok 10900, Thailand; kriskamol.n@ku.ac.th \\ 3 Office of Sericulture Conservation and Standard Conformity Assessment, The Queen Sirikit Department of \\ Sericulture, Ministry of Agriculture and Cooperatives, Bangkok 10900, Thailand; butsara_2000@hotmail.com \\ 4 The Queen Sirikit Department of Sericulture Center (Kanchanaburi), Nong Ya, Mueang Kanchanaburi \\ District, Kanchanaburi 71000, Thailand; yodyingtts@gmail.com \\ 5 Plant Varieties Protection Office, Department of Agriculture, Ministry of Agriculture and Cooperatives, \\ Bangkok 10900, Thailand; kpruesapan@gmail.com \\ 6 Institute of Food Research and Product Development, Kasetsart University, Chatuchak, Bangkok 10900, \\ Thailand; dalad.s@ku.th \\ * Correspondence: uthaiwan.sut@mahidol.ac.th; Tel.: +662-800-2380 (ext. 422)
}

Academic Editors: Jianbo Xiao, Yanbo Zhang and Hui Ni

Received: 6 May 2020; Accepted: 1 June 2020; Published: 3 June 2020

check for updates

\begin{abstract}
Alzheimer's disease (AD) is a progressive neurodegenerative disorder. To fight the disease, natural products, including mulberry, with antioxidant activities and inhibitory activities against key enzymes (acetylcholinesterase (AChE), butyrylcholinesterase (BChE), and beta-secretase 1 (BACE-1)) are of interest. However, even in the same cultivars, mulberry trees grown in different populated locations might possess disparate amounts of phytochemical profiles, leading to dissimilar health properties, which cause problems when comparing different cultivars of mulberry. Therefore, this study aimed to comparatively investigate the phytochemicals, antioxidant activities, and inhibitory activities against AChE, BChE, and BACE-1, of twenty-seven Morus spp. cultivated in the same planting area in Thailand. The results suggested that Morus fruit samples were rich in phenolics, especially cyanidin, kuromanin, and keracyanin. Besides, the aqueous Morus fruit extracts exhibited antioxidant activities, both in single electron transfer (SET) and hydrogen atom transfer (HAT) mechanisms, while strong inhibitory activities against AD key enzymes were observed. Interestingly, among the twenty-seven Morus spp., Morus sp. code SKSM 810191 with high phytochemicals, antioxidant activities and in vitro anti-AD properties is a promising cultivar for further developed as a potential mulberry resource with health benefits against AD.
\end{abstract}

Keywords: Morus species; anthocyanins; anthocyanidins; beta-secretase 1; antioxidant; anti-Alzheimer properties

\section{Introduction}

Aging is recognized as the irreversible and inevitable loss of physiological integrity, leading to aging-associated disorders, including cardiovascular disease, cancer, metabolic syndrome, 
and neurodegenerative diseases [1]. Further studies have also shown that aging increases the decline of nervous system functions. Alzheimer's disease (AD), a devastating type of dementia, is associated with aging, as approximately $90 \%$ of AD cases are found in individuals older than 65 years. It has been suggested that there will be 131 million AD cases by 2050 [2]. However, there are only five drugs approved for AD treatment, including four cholinesterase inhibitors (tacrine, rivastigmine, galantamine, and donepezil) and one glutaminergic $N$-methyl-D-aspartate (NMDA) receptor antagonist (memantine) [3]. Cholinesterase inhibitors have been shown to inhibit the enzymatic functions of cholinesterases, including acetylcholinesterase (AChE) and butyrylcholinesterase (BChE), resulting in the accumulation of a neurotransmitter, acetylcholine, at the synaptic cleft. Meanwhile, memantine blocks the effects of glutamate, which is over-stimulated in AD patients [3]. In addition, an alternative AD treatment has also been intensively studied. Attention has shed light on the beta-secretase 1 (BACE-1) inhibitors because BACE-1 is an enzyme involved in the formation of amyloid or senile plaques, a hallmark of AD. Intriguingly, several reports have highlighted the effective BACE-1 inhibitory activities from plant extracts, such as ursolic acid and lupeol from Leea indica [4], polymethoxyflavones, 5,7-dimethoxyflavone (DMF), 5,7,4'-trimethoxyflavone (TMF), and 3,5,7,3',4'-pentamethoxyflavone (PMF) from Kaempferia parviflora [5], and moracin derivatives from Morus species [6].

Mulberry fruits in Morus species, belonging to the Moraceae family, are widely distributed in tropical, sub-tropical, and sub-arctic regions, including Europe, Asia, America, and Africa, suggesting that Morus spp. is able to adapt to various varieties of climatic and soil conditions [7]. At present, this genus consisting of twenty-four species and one-hundred varieties is suspected to have different health benefits [7]. Morus spp. has long been cultivated and used for sericulture, food, and folk medicine. Mulberry fruits are low in calories with a sour taste $(\mathrm{pH} \leq 3.5)$ and high in phytochemicals, predominantly anthocyanins [8]. Anthocyanins, a member of flavonoids, are responsible for red or purple pigments in vegetables and fruits. The natural-occurring anthocyanins in the plants are in the form of glycosides (binding to carbohydrate moieties), while those without the carbohydrate moieties are subsequently called anthocyanidins [9]. It has been reported that the distribution of anthocyanins in vegetables and fruits is cyanidin (50\%), delphinidin $(12 \%)$, pelargonidin $(12 \%)$, peonidin (12\%), malvidin $(7 \%)$, and petunidin $(7 \%)$, while the most common form of the glycoside derivative in nature is cyanidin 3-glucoside [10]. Anthocyanins exhibit health benefits against a range of ailments, including oxidation, cancer, anemia, obesity, diabetes, hypertension, and AD activities [8]. Given the good taste and health benefits of Morus spp., the cultivation and consumption of mulberry fruits have been swiftly developed around the word, including Thailand.

There are more than thirty mulberry varieties registered by the Queen Sirikit Department of Sericulture, Ministry of Agriculture and Cooperatives, Thailand. However, only a few cultivars have been investigated for their phytochemical profiles and health benefits. Furthermore, regarding the high adaptation of Morus spp. that can be cultivated in several regions of the world, as mentioned above, the Morus spp. cultivated in different areas, even from the same cultivars, might possess different amounts of phytochemical profiles and health properties, leading to difficulty in comparing these parameters. Therefore, this study was undertaken to evaluate the phytochemical profiles (total phenolic, anthocyanin, and anthocyanidin contents), and health properties (antioxidant and anti-Alzheimer properties) of twenty-seven Morus spp. cultivated in the same planting area in Kanchanaburi province, Thailand. This area belongs to the Morus spp. genetic bank project of the Ministry of Agriculture and Cooperatives, Thailand. This is the first study that has provided comparative and comprehensive data of twenty-seven Morus spp., which could indorse further development of the phytochemical compound rich mulberry resources with potential health benefits against the occurrence of AD. 


\section{Results}

\subsection{Total Phenolic Contents (TPCs), Anthocyanins and Anthocyanidins}

The total phenolic contents (TPCs) of Morus fruit extracts were within the range of 0.37-11.86 mg GAE/g dry weight (DW), with Morus sp. code SKSM 810391 extract exhibiting the highest TPCs, and Morus sp. code SKSM 810391 extract providing the lowest (Table 1). The only anthocyanidin in Morus fruit extracts, as determined by HPLC analysis, was detected as cyanidin (Figure 1) ranging between 41.25 and $2879 \mu \mathrm{g} / \mathrm{g}$ DW (Table 1 and Supplementary Figure S1). Morus sp. code SKSM 810191 extract possessed the highest content of cyanidin, while Morus sp. code SRCM 9809-34 extract exhibited the lowest. However, none was detected in Morus sp. code SRCM 9124-12 extract. Two anthocyanins, including keracyanin (cyanidin 3-glucoside) and kuromain (cyanidin 3-rutinoside) (Figure 1), were detected in the range of 121.3-7588 and 88.50-13566 $\mu \mathrm{g} / \mathrm{g} \mathrm{DW}$, respectively (Table 1 and Supplementary Figure S2). The highest keracyanin and kuromanin contents were detected in Morus sp. code SKSM 810191 extract, while the lowest amount was found in Morus sp. code SRCM 9801-535 extract for keracyanin and Morus 'Nakhon Ratchasima 60' extract for kuromanin. However, neither keracyanin nor kuromanin was detected in Morus sp. code SKSM 040691 extract.

Table 1. Quantification of total phenolic contents (TPCs), anthocyanidin (cyanidin) and anthocyanins (keracyanin and kuromanin) of aqueous extracts of Morus fruit samples.

\begin{tabular}{|c|c|c|c|c|c|}
\hline \multirow[t]{2}{*}{ Order } & \multirow[t]{2}{*}{ Cultivars } & \multirow[t]{2}{*}{$\begin{array}{c}\text { TPCs } \\
\text { (mg GAE/g DW) }\end{array}$} & \multirow{2}{*}{$\begin{array}{c}\begin{array}{c}\text { Anthocyanidin } \\
(\mu \mathrm{g} / \mathrm{g} D W)\end{array} \\
\text { Cyanidin }\end{array}$} & \multicolumn{2}{|c|}{$\begin{array}{c}\text { Anthocyanins } \\
(\mu \mathrm{g} / \mathrm{g} \text { DW) }\end{array}$} \\
\hline & & & & Keracyanin & Kuromanin \\
\hline 1 & Morus 'Krua' & $3.72 \pm 0.18^{n}$ & $347.41 \pm 32.02^{f}$ & $883.55 \pm 62.41^{\mathrm{h}}$ & $1249.03 \pm 108.70 \mathrm{~g}$ \\
\hline 2 & Morus 'Jak' & $2.75 \pm 0.11^{\circ}$ & $45.87 \pm 3.01$ ghi & $235.19 \pm 0.86^{\mathrm{mn}}$ & $213.03 \pm 0.90 \mathrm{mn}$ \\
\hline 3 & Morus 'Pai' & $2.76 \pm 0.10^{\circ}$ & $87.58 \pm 7.58$ ghi & $228.07 \pm 2.80^{\mathrm{mn}}$ & $211.58 \pm 2.91 \mathrm{mn}$ \\
\hline 4 & Morus 'Pai-Ubon' & $3.74 \pm 0.17^{n}$ & $86.50 \pm 6.15$ ghi & $237.32 \pm 0.26^{\mathrm{mn}}$ & $160.36 \pm 1.00 \mathrm{mno}$ \\
\hline 5 & Morus 'Poe' & $3.73 \pm 0.12^{n}$ & $160.11 \pm 8.13$ ghi & $522.74 \pm 1.79^{\mathrm{j}}$ & $905.26 \pm 4.89^{\mathrm{h}}$ \\
\hline 6 & Morus 'Mae Luke On' & $4.08 \pm 0.39^{\mathrm{m}}$ & $61.95 \pm 5.00 \mathrm{ghi}$ & $155.23 \pm 2.71$ no & $194.19 \pm 1.40 \mathrm{mn}$ \\
\hline 7 & Morus 'Som' & $5.18 \pm 0.19 \mathrm{jk}$ & $98.12 \pm 4.18 \mathrm{ghi}$ & $235.99 \pm 5.41 \mathrm{mn}$ & $172.13 \pm 3.05 \mathrm{mn}$ \\
\hline 8 & Morus 'Som Yai' & $4.10 \pm 0.38^{\mathrm{m}}$ & $104.06 \pm 11.16^{\mathrm{ghi}}$ & $299.28 \pm 0.40^{\mathrm{lm}}$ & $263.20 \pm 1.78^{\mathrm{klmn}}$ \\
\hline 9 & Morus 'Sida' & $5.58 \pm 0.48^{\mathrm{hi}}$ & $184.74 \pm 16.86^{\mathrm{gh}}$ & $630.07 \pm 1.09^{\mathrm{i}}$ & $814.19 \pm 1.41^{\mathrm{h}}$ \\
\hline 10 & Morus 'Kun Pai' & $7.87 \pm 0.43^{\mathrm{e}}$ & $1710.50 \pm 155.12^{b c}$ & $2765.88 \pm 42.49^{\mathrm{d}}$ & $7374.67 \pm 30.48^{c}$ \\
\hline 11 & Morus 'Nakhon Ratchasima 60' & $3.53 \pm 0.20^{n}$ & $48.95 \pm 5.08$ ghi & $161.99 \pm 0.30^{\text {no }}$ & $88.50 \pm 0.38^{\text {no }}$ \\
\hline 12 & Morus 'Buri Ram 51' & $3.70 \pm 0.29^{n}$ & $107.50 \pm 8.29$ ghi & $536.22 \pm 37.80^{j}$ & $222.42 \pm 18.22 \mathrm{mn}$ \\
\hline 13 & Morus 'Buri Ram 60' & $4.68 \pm 0.23^{1}$ & $68.23 \pm 0.23$ ghi & $372.89 \pm 0.03^{\mathrm{kl}}$ & $309.09 \pm 0.78 \mathrm{klm}$ \\
\hline 14 & Morus 'Si Sa Ket 33' & $5.45 \pm 0.44^{\mathrm{ij}}$ & $43.16 \pm 4.92 \mathrm{hi}$ & $498.91 \pm 0.92^{j}$ & $405.97 \pm 1.35^{\mathrm{jkl}}$ \\
\hline 15 & Morus 'Number $44^{\prime}$ & $8.84 \pm 0.74^{\mathrm{d}}$ & $143.25 \pm 11.45$ ghi & $1025.16 \pm 5.36^{g}$ & $1233.66 \pm 19.54 \mathrm{~g}$ \\
\hline 16 & Morus sp. code SKSM 820281 & $7.11 \pm 0.58^{f}$ & $1789.96 \pm 127.59 \mathrm{ab}$ & $4874.70 \pm 83.40^{c}$ & $6426.53 \pm 156.12^{d}$ \\
\hline 17 & Morus sp. code SKSM 14-13-20 & $10.81 \pm 0.21^{b}$ & $1583.49 \pm 113.87^{\mathrm{cd}}$ & $5848.59 \pm 65.45^{b}$ & $10141.24 \pm 71.43^{b}$ \\
\hline 18 & Morus sp. code SKSM 040691 & $5.05 \pm 0.28^{k}$ & $62.07 \pm 6.66$ ghi & $0.00 \mathrm{P}$ & $0.00^{\circ}$ \\
\hline 19 & Morus sp. code SKSM 810191 & $10.27 \pm 0.50^{c}$ & $2879.30 \pm 228.33^{a}$ & $7588.34 \pm 36.59^{a}$ & $13566.64 \pm 37.40^{\mathrm{a}}$ \\
\hline 20 & Morus sp. code SKSM 810391 & $0.37 \pm 0.01 \mathrm{P}$ & $1502.84 \pm 157.69^{\mathrm{d}}$ & $2408.50 \pm 159.13^{\mathrm{e}}$ & $5447.20 \pm 305.14^{\mathrm{e}}$ \\
\hline 21 & Morus sp. code SRCM 9124-12 & $5.81 \pm 0.19^{h}$ & $0.00^{\mathrm{i}}$ & $403.03 \pm 0.92^{k}$ & $560.18 \pm 2.29^{\mathrm{ij}}$ \\
\hline 22 & Morus sp. code SRCM 9801-465 & $7.20 \pm 0.48^{\mathrm{f}}$ & $182.72 \pm 14.62 \mathrm{gh}$ & $720.44 \pm 61.74^{i}$ & $1363.68 \pm 111.80^{g}$ \\
\hline 23 & Morus sp. code SRCM 9801-535 & $5.58 \pm 0.28$ hi & $207.84 \pm 10.43 \mathrm{fg}$ & $121.33 \pm 8.63^{\circ}$ & $139.19 \pm 9.84 \mathrm{mno}$ \\
\hline 24 & Morus sp. code SRCM 9801-833 & $5.39 \pm 0.29^{i j}$ & $344.58 \pm 29.88^{f}$ & $346.69 \pm 5.19 \mathrm{kl}$ & $421.43 \pm 6.22 \mathrm{jk}$ \\
\hline 25 & Morus sp. code SRCM 9806-271 & $6.79 \pm 0.20 \mathrm{~g}$ & $713.78 \pm 25.11 \mathrm{e}$ & $1428.44 \pm 0.07^{\mathrm{f}}$ & $2634.51 \pm 9.53^{f}$ \\
\hline 26 & Morus sp. code SRCM 9806-287 & $11.86 \pm 0.19^{a}$ & $676.62 \pm 41.02 e^{e}$ & $685.19 \pm 11.64^{i}$ & $611.59 \pm 8.34^{\mathrm{i}}$ \\
\hline 27 & Morus sp. code SRCM 9809-34 & $5.55 \pm 0.26^{\mathrm{hi}}$ & $41.25 \pm 1.68^{\mathrm{hi}}$ & $152.75 \pm 1.02^{\text {no }}$ & $247.17 \pm 0.20^{l m n}$ \\
\hline
\end{tabular}

Values expressed are mean \pm standard deviation (SD) of triplicate experiments $(n=3)$. Lowercase letter indicates significant differences in each column at $p<0.05$ calculated by one-way analysis of variance (ANOVA) and Duncan's multiple comparison test.

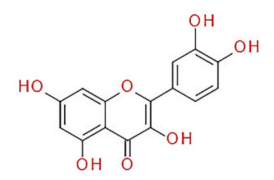

A.

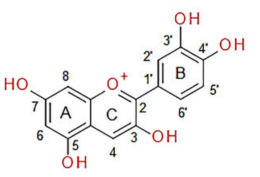

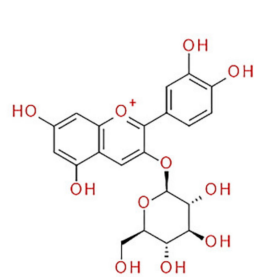

C.

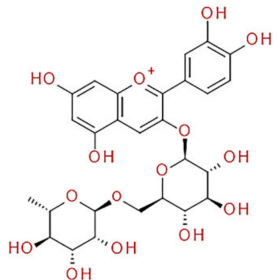

D.

Figure 1. Chemical structures of (A) quercetin, (B) cyanidin, (C) kuromanin (cyanidin 3-glucoside), and (D) keracyanin (cyanidin 3-rutinoside). 


\subsection{Antioxidant Activities}

Antioxidant activities of Morus fruit extracts were determined using 2,2-diphenyl-1-picrylhydrazyl (DPPH) radical scavenging activity, ferric ion reducing antioxidant power (FRAP), and oxygen radical absorbance capacity (ORAC) assays. The difference among these methods involved the reaction mechanisms, in which DPPH radical scavenging and FRAP assays presented the mechanism of single electron transfer (SET), while ORAC assay underwent the hydrogen atom transfer (HAT) mechanism [11]. Since various species of oxidants (i.e., reactive oxygen species and reactive nitrogen species) were generated, more than one assay for detecting antioxidant activities was suggested to appropriately interpret the antioxidant results.

The results (Table 2) suggested that all Morus fruit extracts exhibited scavenging activities of 0.28-1.25 $\mu \mathrm{mol}$ TE/100 g DW with Morus 'Kun Pai' extract exhibiting the highest activity and Morus 'Nakhon Ratchasima 60' extract the lowest. The chelating abilities of ferrous ion ranged between 2.30 and $117.8 \mu \mathrm{mol}$ TE/g DW, as investigated by the FRAP assay. The highest chelating ability was found in Morus sp. code SKSM 810391 extract, while the lowest was in Morus 'Pai' extract. Antioxidant capacity measured by the ORAC assay ranged between 64.03 and $283.2 \mu \mathrm{mol}$ TE/g DW. Morus sp. code SKSM 810191 extract possessed the highest ORAC activity, while Morus sp. code SRCM 9124-12 extract exhibited the lowest.

Table 2. Antioxidant analysis of aqueous extracts of Morus fruit samples.

\begin{tabular}{|c|c|c|c|c|}
\hline Order & Cultivars & $\begin{array}{c}\text { DPPH Radical } \\
\text { Scavenging Assay } \\
(\mu \mathrm{mol} \text { TE/100 g DW })\end{array}$ & $\begin{array}{c}\text { FRAP Assay } \\
(\mu \mathrm{mol} \mathrm{TE} / \mathrm{g} \text { DW })\end{array}$ & $\begin{array}{c}\text { ORAC Assay } \\
(\mu \mathrm{mol} \text { TE } / \mathrm{g} \text { DW })\end{array}$ \\
\hline 1 & Morus 'Krua' & $0.62 \pm 0.05^{\mathrm{e}}$ & $4.39 \pm 0.18^{\mathrm{m}}$ & $201.81 \pm 15.60^{\mathrm{cd}}$ \\
\hline 2 & Morus 'Jak' & $0.50 \pm 0.05 \mathrm{gh}$ & $2.45 \pm 0.19^{n}$ & $134.19 \pm 11.06^{\mathrm{gh}}$ \\
\hline 3 & Morus 'Pai' & $0.49 \pm 0.05^{h}$ & $2.30 \pm 0.17^{n}$ & $151.04 \pm 13.62 \mathrm{fg}$ \\
\hline 4 & Morus 'Pai-Ubon' & $0.56 \pm 0.05^{f}$ & $2.61 \pm 0.11 \mathrm{mn}$ & $172.53 \pm 15.75$ ef \\
\hline 5 & Morus 'Poe' & $0.58 \pm 0.05^{\mathrm{f}}$ & $3.33 \pm 0.16^{\mathrm{mn}}$ & $151.16 \pm 12.03 \mathrm{fg}$ \\
\hline 6 & Morus 'Mae Luke On' & $0.45 \pm 0.04^{h}$ & $14.24 \pm 0.84^{\mathrm{jk}}$ & $130.52 \pm 12.01 \mathrm{ghi}$ \\
\hline 7 & Morus 'Som & $0.45 \pm 0.04^{h}$ & $18.50 \pm 0.79^{h}$ & $254.04 \pm 23.15^{b}$ \\
\hline 8 & Morus 'Som Yai' & $0.50 \pm 0.04 \mathrm{gh}$ & $13.32 \pm 0.63^{k}$ & $91.92 \pm 8.78 \mathrm{klm}$ \\
\hline 9 & Morus 'Sida' & $0.54 \pm 0.05 \mathrm{fg}$ & $16.55 \pm 1.43^{\mathrm{i}}$ & $112.16 \pm 9.24^{\text {hijk }}$ \\
\hline 10 & Morus 'Kun Pai' & $1.25 \pm 0.02^{\mathrm{a}}$ & $44.33 \pm 0.76^{d}$ & $216.42 \pm 53.27^{c}$ \\
\hline 11 & Morus 'Nakhon Ratchasima 60' & $0.28 \pm 0.02^{k}$ & $11.02 \pm 0.54^{1}$ & $131.73 \pm 12.37 \mathrm{ghi}$ \\
\hline 12 & Morus 'Buri Ram 51' & $0.32 \pm 0.03^{j}$ & $11.37 \pm 0.47^{1}$ & $103.07 \pm 6.55^{\mathrm{jkl}}$ \\
\hline 13 & Morus 'Buri Ram 60' & $0.37 \pm 0.03^{i}$ & $15.50 \pm 0.90 \mathrm{ij}$ & $158.20 \pm 11.01^{\mathrm{f}}$ \\
\hline 14 & Morus 'Si Sa Ket 33' & $0.37 \pm 0.01^{\mathrm{i}}$ & $15.56 \pm 1.33$ ij & $178.98 \pm 17.51^{\mathrm{e}}$ \\
\hline 15 & Morus 'Number 44' & $0.83 \pm 0.08^{b}$ & $27.90 \pm 1.10^{g}$ & $251.84 \pm 21.98^{b}$ \\
\hline 16 & Morus sp. code SKSM 820281 & $0.50 \pm 0.03 \mathrm{gh}$ & $43.28 \pm 2.85^{d}$ & $192.66 \pm 48.44 \mathrm{de}$ \\
\hline 17 & Morus sp. code SKSM 14-13-20 & $0.72 \pm 0.05^{c}$ & $66.96 \pm 6.48^{b}$ & $259.25 \pm 40.32^{b}$ \\
\hline 18 & Morus sp. code SKSM 040691 & $0.67 \pm 0.06^{\mathrm{d}}$ & $14.70 \pm 0.20^{\mathrm{ijk}}$ & $86.63 \pm 7.72 \mathrm{~lm}$ \\
\hline 19 & Morus sp. code SKSM 810191 & $0.75 \pm 0.04^{c}$ & $63.97 \pm 3.84^{c}$ & $283.20 \pm 36.56^{\mathrm{a}}$ \\
\hline 20 & Morus sp. code SKSM 810391 & $0.75 \pm 0.07^{c}$ & $117.87 \pm 1.77^{\mathrm{a}}$ & $109.74 \pm 3.644^{\mathrm{ijk}}$ \\
\hline 21 & Morus sp. code SRCM 9124-12 & $0.73 \pm 0.06^{c}$ & $15.75 \pm 0.53^{\mathrm{ij}}$ & $64.03 \pm 4.32^{n}$ \\
\hline 22 & Morus sp. code SRCM 9801-465 & $0.55 \pm 0.03^{f}$ & $3.06 \pm 0.23 \mathrm{mn}$ & $77.71 \pm 3.84 \mathrm{mn}$ \\
\hline 23 & Morus sp. code SRCM 9801-535 & $0.56 \pm 0.05^{f}$ & $16.64 \pm 3.17^{\mathrm{i}}$ & $103.79 \pm 8.10^{\mathrm{jkl}}$ \\
\hline 24 & Morus sp. code SRCM 9801-833 & $0.55 \pm 0.04^{\mathrm{f}}$ & $14.20 \pm 0.71^{j \mathrm{k}}$ & $113.79 \pm 9.44^{\text {hijk }}$ \\
\hline 25 & Morus sp. code SRCM 9806-271 & $0.67 \pm 0.06^{\mathrm{d}}$ & $30.15 \pm 1.52^{f}$ & $151.52 \pm 11.82 \mathrm{fg}$ \\
\hline 26 & Morus sp. code SRCM 9806-287 & $0.85 \pm 0.07^{b}$ & $40.52 \pm 1.16^{\mathrm{e}}$ & $116.96 \pm 9.64$ hij \\
\hline 27 & Morus sp. code SRCM 9809-34 & $0.55 \pm 0.03^{f}$ & $15.28 \pm 1.34^{\mathrm{ij}}$ & $82.85 \pm 5.84^{1 \mathrm{mn}}$ \\
\hline
\end{tabular}

Values expressed are mean \pm standard deviation (SD) of triplicate experiments $(n=3)$. Lowercase letter indicates significant differences in each column at $p<0.05$ calculated by one-way analysis of variance (ANOVA) and Duncan's multiple comparison test.

\subsection{In Vitro Cholinesterase and BACE-1 Inhibitory Activities}

Morus fruit extracts were able to inhibit the key enzymes involved in AD, including AChE, BChE, and BACE-1, with different degrees of inhibition (Table 3). The AChE inhibitory activities of all Morus fruit extracts were in the range of $21.87-60.09 \%$ inhibition at the extract concentration of $5 \mathrm{mg} / \mathrm{mL}$. 
Morus 'Nakhon Ratchasima 60' extract exhibited the highest AChE inhibitory activity, while Morus sp. code SKSM 810391 extract exhibited the lowest. Under the same extract concentration, BChE inhibitory activities ranged between $21.27 \%$ and $77.02 \%$ inhibition, with Morus 'Som' extract exhibiting the highest inhibition and Morus sp. code SRCM 9801-465 extract the lowest. Likewise, Morus fruit extracts exhibited BACE-1 inhibitory activity in the range of $31.28-78.67 \%$ inhibition at the extract concentration of $5 \mathrm{mg} / \mathrm{mL}$. Morus sp. code SRCM 9806-287 extract exhibited the highest BACE-1 inhibition, while the lowest inhibition was detected in Morus sp. code SRCM 9124-12 extract.

Table 3. Anti-Alzheimer properties of aqueous extracts of Morus fruit samples towards inhibitions of acetylcholinesterase (AChE), butyrylcholinesterase (BChE), and beta-secretase 1 (BACE-1).

\begin{tabular}{|c|c|c|c|c|}
\hline \multirow{2}{*}{ Order } & \multirow{2}{*}{ Cultivars } & \multicolumn{3}{|c|}{ Percentage of Inhibition (\%) } \\
\hline & & AChE & BChE & BACE-1 \\
\hline 1 & Morus 'Krua' & $44.91 \pm 2.62^{f}$ & $42.38 \pm 2.35^{j}$ & $41.58 \pm 7.89^{j}$ \\
\hline 2 & Morus 'Jak' & $37.35 \pm 1.54 \mathrm{ij}$ & $51.16 \pm 4.58 \mathrm{~g}$ & $58.61 \pm 0.41$ efg \\
\hline 3 & Morus 'Pai' & $34.68 \pm 3.11^{\mathrm{jkl}}$ & $51.30 \pm 3.62 \mathrm{~g}$ & $38.78 \pm 2.26^{j}$ \\
\hline 4 & Morus 'Pai-Ubon' & $37.09 \pm 3.44^{\mathrm{ijk}}$ & $48.36 \pm 3.63 \mathrm{gh}$ & $71.23 \pm 0.00 \mathrm{bc}$ \\
\hline 5 & Morus 'Poe' & $33.70 \pm 3.31 \mathrm{~lm}$ & $55.34 \pm 1.05^{\mathrm{f}}$ & $37.48 \pm 2.07 \mathrm{jk}$ \\
\hline 6 & Morus 'Mae Luke On' & $45.71 \pm 3.95$ ef & $56.52 \pm 5.16^{\mathrm{ef}}$ & $51.27 \pm 3.32$ ghi \\
\hline 7 & Morus 'Som' & $56.29 \pm 2.41^{b}$ & $77.02 \pm 3.14^{\mathrm{a}}$ & $63.01 \pm 5.22$ de \\
\hline 8 & Morus 'Som Yai' & $53.89 \pm 4.20 \mathrm{bc}$ & $63.16 \pm 1.03^{c}$ & $63.00 \pm 7.50$ de \\
\hline 9 & Morus 'Sida' & $49.65 \pm 2.53^{\mathrm{d}}$ & $64.86 \pm 2.39^{c}$ & $52.81 \pm 1.37$ ghi \\
\hline 10 & Morus 'Kun Pai' & $35.37 \pm 1.77^{\mathrm{jkl}}$ & $41.78 \pm 1.28^{j}$ & $48.93 \pm 1.66^{\mathrm{i}}$ \\
\hline 11 & Morus 'Nakhon Ratchasima 60' & $60.09 \pm 3.62^{a}$ & $62.72 \pm 5.23^{c}$ & $54.21 \pm 2.69$ ghi \\
\hline 12 & Morus 'Buri Ram 51' & $53.66 \pm 3.40^{b c}$ & $71.33 \pm 6.79^{b}$ & $65.54 \pm 3.44^{\text {cde }}$ \\
\hline 13 & Morus 'Buri Ram 60' & $45.61 \pm 3.96^{\text {ef }}$ & $56.85 \pm 1.50$ ef & $70.65 \pm 1.02 \mathrm{bc}$ \\
\hline 14 & Morus 'Si Sa Ket 33' & $45.85 \pm 4.47^{\text {ef }}$ & $62.99 \pm 3.03^{c}$ & $76.32 \pm 2.06^{\mathrm{ab}}$ \\
\hline 15 & Morus 'Number 44' & $50.98 \pm 2.73^{\mathrm{cd}}$ & $61.31 \pm 3.53^{\mathrm{cd}}$ & $70.45 \pm 3.54 \mathrm{bc}$ \\
\hline 16 & Morus sp. code SKSM 820281 & $34.05 \pm 3.70 \mathrm{klm}$ & $48.76 \pm 4.59 \mathrm{gh}$ & $55.12 \pm 1.38$ fghi \\
\hline 17 & Morus sp. code SKSM 14-13-20 & $31.37 \pm 2.25^{\mathrm{m}}$ & $37.80 \pm 3.79 \mathrm{k}$ & $77.11 \pm 5.60^{\mathrm{ab}}$ \\
\hline 18 & Morus sp. code SKSM 040691 & $26.10 \pm 1.44^{\mathrm{n}}$ & $43.86 \pm 0.91 \mathrm{ij}$ & $54.60 \pm 3.59$ fghi \\
\hline 19 & Morus sp. code SKSM 810191 & $43.68 \pm 2.28 \mathrm{fg}$ & $59.05 \pm 2.28 \mathrm{de}$ & $66.34 \pm 2.06^{\mathrm{cd}}$ \\
\hline 20 & Morus sp. code SKSM 810391 & $21.87 \pm 1.48^{\circ}$ & $22.02 \pm 2.20 \mathrm{~m}$ & $61.64 \pm 6.11$ def \\
\hline 21 & Morus sp. code SRCM 9124-12 & $48.52 \pm 4.78 \mathrm{de}$ & $50.57 \pm 2.22 \mathrm{gh}$ & $31.28 \pm 1.78^{\mathrm{k}}$ \\
\hline 22 & Morus sp. code SRCM 9801-465 & $22.96 \pm 1.42^{\circ}$ & $21.27 \pm 1.96^{\mathrm{m}}$ & $58.10 \pm 3.23$ efgh \\
\hline 23 & Morus sp. code SRCM 9801-535 & $39.98 \pm 2.72 \mathrm{hi}$ & $61.63 \pm 3.07^{\mathrm{cd}}$ & $51.03 \pm 0.36 \mathrm{hi}$ \\
\hline 24 & Morus sp. code SRCM 9801-833 & $36.50 \pm 2.07^{\mathrm{jkl}}$ & $48.75 \pm 1.48 \mathrm{gh}$ & $62.85 \pm 0.97 \mathrm{de}$ \\
\hline 25 & Morus sp. code SRCM 9806-271 & $41.21 \pm 3.00 \mathrm{gh}$ & $51.87 \pm 4.24^{\mathrm{g}}$ & $76.12 \pm 5.45^{\mathrm{ab}}$ \\
\hline 26 & Morus sp. code SRCM 9806-287 & $26.63 \pm 2.20^{n}$ & $30.00 \pm 2.60^{1}$ & $78.67 \pm 5.70^{\mathrm{a}}$ \\
\hline 27 & Morus sp. code SRCM 9809-34 & $36.17 \pm 3.55^{\mathrm{jkl}}$ & $47.06 \pm 3.21 \mathrm{hi}$ & $64.79 \pm 2.52^{\text {cde }}$ \\
\hline
\end{tabular}

Values expressed are mean \pm standard deviation (SD) of triplicate experiments $(n=3)$. Lowercase letter indicates significant differences in each column at $p<0.05$ calculated by one-way analysis of variance (ANOVA) and Duncan's multiple comparison test. The extract concentration was $5 \mathrm{mg} / \mathrm{mL}$ in all enzyme assays.

\subsection{Correlation Analysis of Bioactive Compounds, Antioxidant Activities and AD Key Enzyme Inhibitory Activities}

The relationship among bioactive compounds, antioxidant activities and enzyme inhibitory activities was reported as correlation coefficients $(r)$ between values (Table 4$)$. The $r$ ranges were divided into three groups; weak correlation $(r=-/+0.10$ to $-/+0.29)$, moderate correlation $(r=-/+0.30$ to $-/+0.49)$ and strong correlation $(r=-/+0.50$ to $-/+1.0)$. The results among phytochemical compounds suggested a strong positive correlation between TPCs and the contents of keracyanin $(r=0.540)$ and kuromanin $(r=0.505)$, while a moderate positive correlation between TPCs and cyanidin contents was detected $(r=0.476)$. Moreover, strong positive correlations among cyanidin, keracyanin and kuromanin were observed ( $r=0.943-0.977)$. The relationship between phytochemical compounds and antioxidant activities suggested a strong positive correlation between TPCs and DPPH radical scavenging activities $(r=0.502)$, while forming a moderate positive correlation with ORAC activities $(r=0.421)$ and a 
weak positive correlation with FRAP activities $(r=0.242)$. On the other hand, keracyanin formed strong positive correlations with antioxidant activities determined by FRAP $(r=0.678)$ and ORAC assays $(r=0.626)$. Similar results were observed with strong positive correlations between cyanidin and kuromanin with all three methods of antioxidant measurement $(r=0.527-0.772$ for cyanidin and 0.498-0.725 for kuromanin). Nevertheless, weak to moderate correlations between the amounts of phytochemicals compounds and inhibitory activities of AD key enzymes were observed with $r$ ranging from -0.305 to 0.416 . Likewise, weak to moderate correlations between antioxidant activities and inhibitory activities of AD key enzymes were reported with $r$ ranging from -0.480 to 0.300 . Interestingly, AChE inhibitory activities formed a strong correlation $(r=0.860)$ with BChE inhibitory activities, while no correlations were observed between inhibitory activities of cholinesterase and BACE-1 $(r$ ranged from -0.037 to -0.020$)$.

To independently analyze the correlation among bioactive compounds, antioxidant activities and enzyme inhibitory activities, mean values of all variables, including phenolic contents (TPCs, cyanidin, keracyanin, and kuromanin), antioxidant activities (DPPH radical scavenging, FRAP, and ORAC values), and inhibitory activities of AD key enzymes (AChE, BChE, and BACE-1), obtained for twenty-seven Morus cultivars were subjected to statistical analysis via principal component analysis (PCA) to verify if the mulberry cultivars could be differentiated according to the mentioned variables. A PCA biplot (Figure 2) showed that differentiation among twenty-seven Morus cultivars shifted along the PC1 and PC2 axes representing $\sim 65 \%$ of total variables. TPCs, anthocyanins, anthocyanidin, antioxidant activities and BACE-1 inhibitory activities were clustering together. Furthermore, agglomerative hierarchical clustering analysis (AHC) in similarity mode was performed as shown in Figure 3 and resulted in twenty-seven Morus cultivars being divided into four groups. Interestingly, both PCA biplot and the dendrogram showed that a group of six cultivars, including Morus sp. code SKSM 810191, SKSM 810391, SKSM 820281, SKSM 14-13-20, SRCM 9806-287 and Morus 'Kun Pai', was gathered together based on their high phytochemicals, antioxidant activities and BACE-1 inhibitory activities.

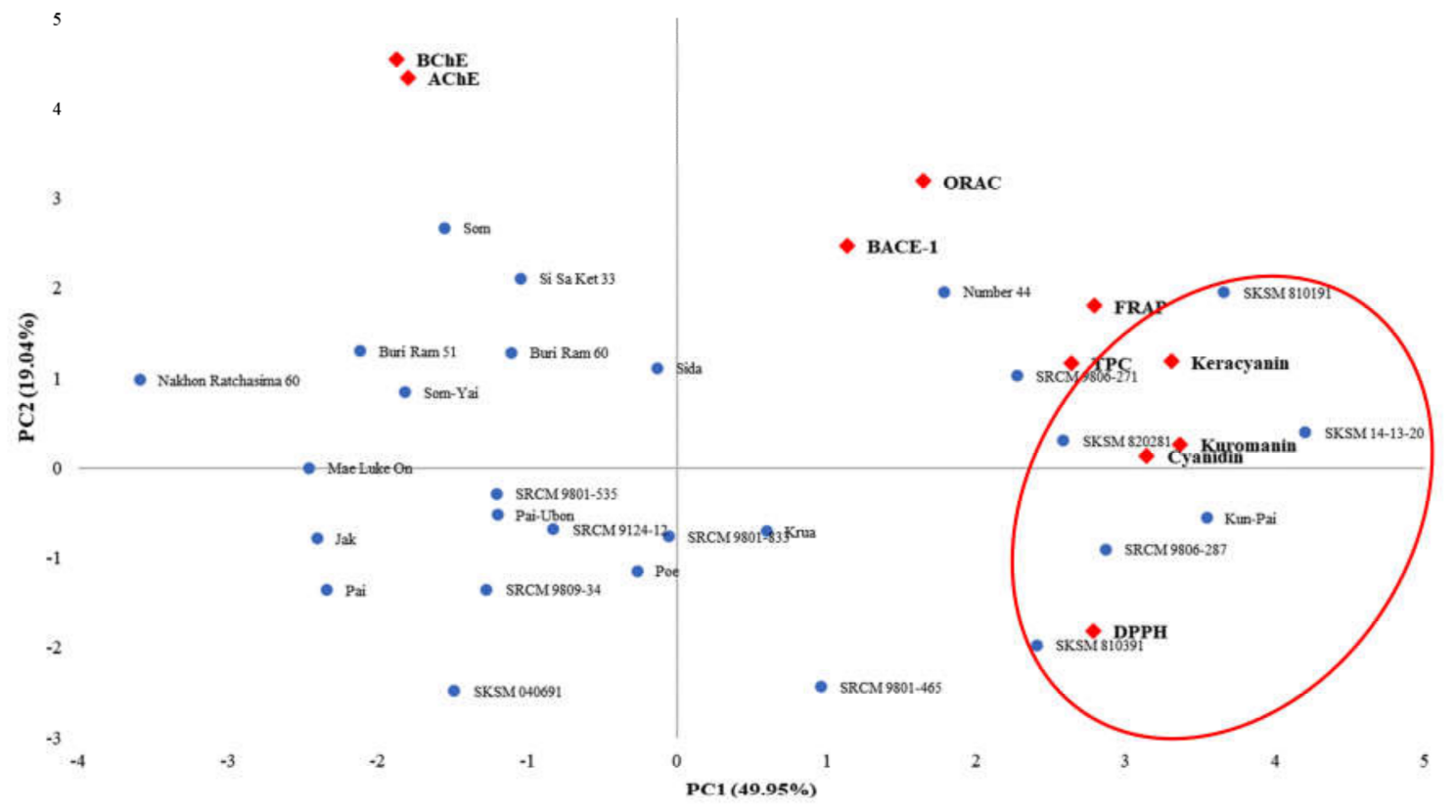

Figure 2. Biplot of principal component analysis from mean value of all variables $(\bullet)$ investigated in twenty-seven Morus cultivars ( $\bullet$ ). 
Table 4. Correlation coefficient ( $r$ ) of total phenolic contents (TPCs), cyanidin contents, keracyanin contents, kuromanin contents, antioxidant activities as being determined by 2,2-diphenyl-1-picrylhydrazyl (DPPH) radical scavenging, ferric ion reducing antioxidant power (FRAP), and oxygen radical absorbance capacity (ORAC) assays and anti-Alzheimer activities through inhibition of the key enzymes (acetylcholinesterase (AChE), butyrylcholinesterase (BChE), and beta-secretase 1 (BACE-1)) of aqueous extracts of Morus fruit samples.

\begin{tabular}{|c|c|c|c|c|c|c|c|c|c|c|}
\hline Parameters & TPCs & Cyanidin & Keracyanin & Kuromanin & DPPH & FRAP & ORAC & Anti-AChE & Anti-BChE & Anti-BACE1 \\
\hline TPCs & 1 & & & & & & & & & \\
\hline Cyanidin & 0.476 * & 1 & & & & & & & & \\
\hline Keracyanin & $0.540^{* *}$ & $0.943^{* *}$ & 1 & & & & & & & \\
\hline Kuromanin & $0.505^{* *}$ & $0.963^{* *}$ & $0.977^{* *}$ & 1 & & & & & & \\
\hline DPPH & $0.502 * *$ & 0.527 ** & 0.381 & $0.498^{* *}$ & 1 & & & & & \\
\hline FRAP & 0.242 & 0.772 ** & $0.678^{* *}$ & $0.725^{* *}$ & $0.481 *$ & 1 & & & & \\
\hline ORAC & 0.421 * & 0.543 ** & $0.626^{* *}$ & $0.610^{* *}$ & 0.269 & 0.300 & 1 & & & \\
\hline Anti-AChE & -0.138 & -0.305 & -0.204 & -0.247 & -0.418 * & -0.360 & 0.178 & 1 & & \\
\hline Anti-BChE & -0.154 & -0.297 & -0.193 & -0.252 & -0.480 * & -0.413 * & 0.208 & 0.860 ** & 1 & \\
\hline Anti-BACE1 & $0.416^{*}$ & 0.178 & 0.213 & 0.175 & -0.350 & 0.300 & 0.269 & -0.037 & -0.020 & 1 \\
\hline
\end{tabular}

** Correlation is significant at $p \leq 0.01$ (2-tailed bivariated correlation). ${ }^{*}$ Correlation is significant at $p \leq 0.05$ (2-tailed bivariated correlation). 


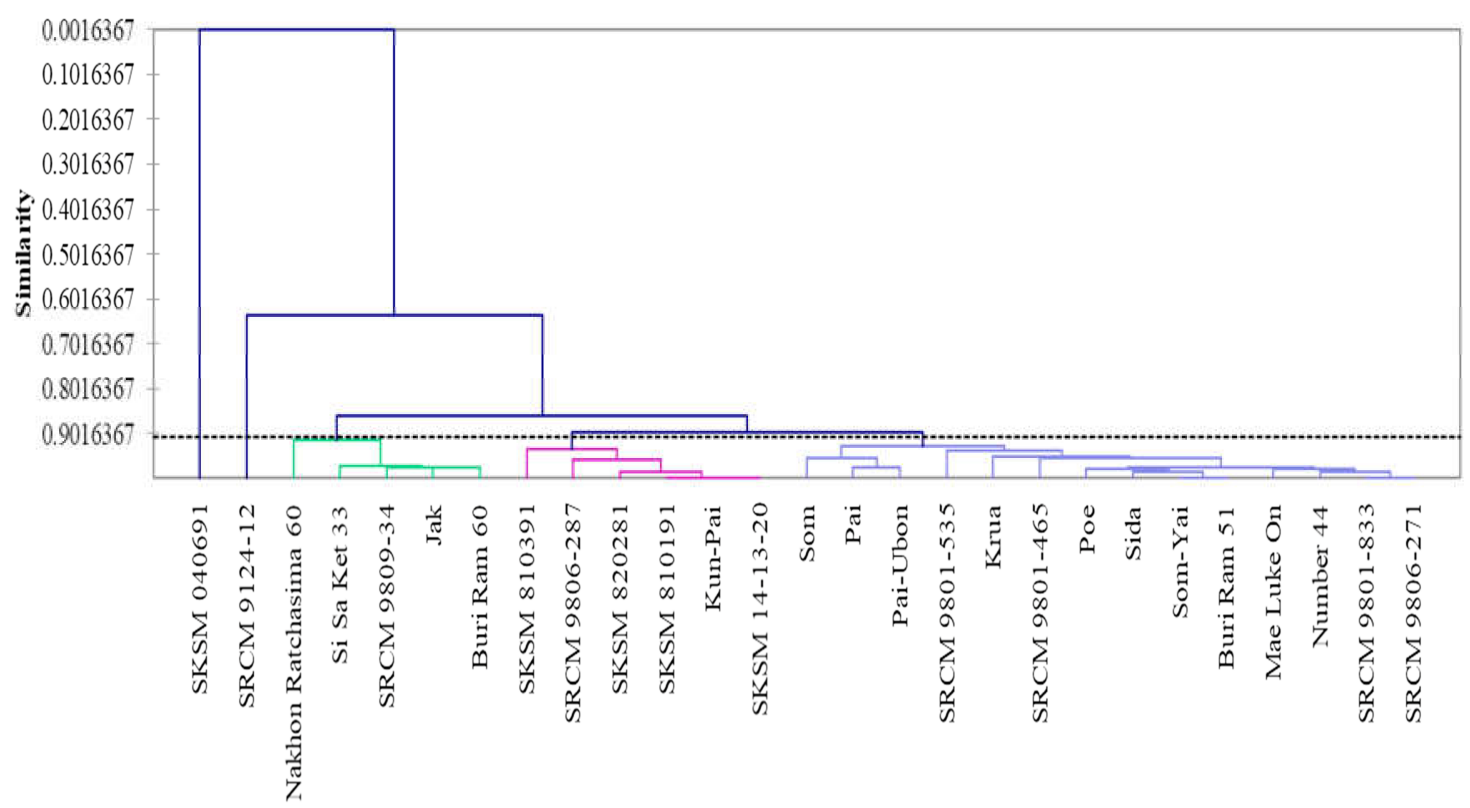

Figure 3. Dendrogram (similarity mode of agglomerative hierarchical clustering analysis) of twenty-seven Morus cultivars by mean value of all variables.

\section{Discussion}

There is evidence demonstrating that plant extracts possess potential health benefits against $\mathrm{AD}$ through several mechanisms underlying AD pathogenesis, particularly by the reduction of oxidative stress, cholinesterases, and BACE- 1 activities. In addition to their efficacy, plant extracts also seem to be safer than synthetic drugs. Morus spp. are of great interest due to their high phytochemicals, especially anthocyanins and anthocyanidins, which have been proved to exhibit anti-AD functions in vitro and in vivo [12]. Besides anthocyanins and anthocyanidins, moracin derivatives from Morus radix could function as dual BACE1 and cholinesterase inhibitors with antioxidant and anti-glycation capacities [6]. In Thailand, more than thirty mulberry varieties are planted under the Morus spp. genetic bank project of the Ministry of Agriculture and Cooperatives. However, the phytochemical profiles and health properties of these mulberry fruits are still missing. Therefore, this is the first comparative and comprehensive study of twenty-seven Morus spp. regarding their phytochemical profiles (total phenolic contents, as well as anthocyanins and anthocyanidins contents), and health properties (anti-oxidant and anti-Alzheimer properties). We have found that (i) Morus fruit samples were rich in phenolics, anthocyanidin (cyanidin) and anthocyanins (kuromanin and keracyanin), (ii) aqueous Morus fruit extracts are involved in anti-oxidative stress, both in single electron transfer (SET) and hydrogen atom transfer (HAT) mechanisms, (iii) aqueous Morus fruit extracts exhibited strong inhibitory activities against AD key enzymes (AChE, BChE and BACE-1), and (vi) Morus sp. code SKSM 810191 provided high TPCs, anthocyanins and anthocyanidin contents, antioxidant activities and in vitro anti-AD properties, which can be further developed as a potential mulberry resource with health benefits against $\mathrm{AD}$.

It was previously suggested that colors (cultivars) of mulberry yielded a great impact on their bioactive compounds [7,13-15]. Juice of white (M. alba L.), red (M. rubra L.), and black (M. nigra L.) mulberry fruits from Turkey suggested that black mulberry exhibited the highest TPCs (1422 mg GAE/100 $\mathrm{g}$ fresh weight (FW)), followed by red (1035 mg GAE/100 $\mathrm{g} \mathrm{FW)} \mathrm{and} \mathrm{white} \mathrm{mulberries} \mathrm{(181} \mathrm{mg}$ GAE/100 g FW), respectively [7]. These data corresponded to our study, in which the TPCs of the aqueous Morus fruit extracts (purple-red color) ranged between 71 and $2270 \mathrm{mg}$ GAE/100 FW (or 0.37-11.86 mg GAE/g DW). Besides, the TPCs of the aqueous ethanolic extracted M. alba from Korea (the TPCs of 0.96-2.57 mg GAE/g DW) [16] and the methanolic extracted M. alba from North Serbia (the TPCs of 1.05-2.16 mg GAE/g DW) [17] were in the same range as the TPCs of our aqueous Morus fruit extracts 
(0.37-11.86 mg GAE/g DW). As for anthocyanidins, cyanidin is the most abundant anthocyanidin (50\%) detected in fruits and vegetables, followed by delphinidin $(12 \%)$, pelargonidin $(12 \%)$, peonidin $(12 \%)$, malvidin (7\%), and petunidin (7\%) [18]. Cyanidin gives a magenta color; thus, it is mostly found in reddish-purple berries or vegetables [19]. In our experiment, cyanidin (41.25-2879.30 $\mu \mathrm{g} / \mathrm{g}$ DW) was the only anthocyanidin detected in all Morus fruit extracts, while delphinidin, pelargonidin, peonidin and petunidin were undetected. Besides, two glycosides of cyanidin, keracyanin (cyanidin 3-rutinoside, 121.3-7,588 $\mu \mathrm{g} / \mathrm{g} \mathrm{DW}$,) and kuromanin (cyanidin 3-glucoside, 88.50-13,566 $\mu \mathrm{g} / \mathrm{g} \mathrm{DW}$ ), were found in our Morus fruit extracts. These results corresponded to the previous studies, which suggested that the predominant anthocyanins found in mulberry ( $M$. alba L.) extracted with acidic ethanol were keracyanin $(60 \%)$ and kuromain $(38 \%)$, while traces of pelargonidin 3-O-glucoside and pelargonidin 3 -O-rutinoside were also detected at $2 \%$ in total [20]. Previous studies also suggested that aqueous ethanolic extracts of five cultivars of Korean mulberry (M. alba L.) exhibited keracyanin, ranging between 30.6 and $486.7 \mu \mathrm{g} / \mathrm{g}$ DW, and kuromanin ranging between 93.2 and $1364.9 \mu \mathrm{g} / \mathrm{g}$ DW [16], which were in the ranges of our anthocyanin contents. Other than these major anthocyanins, Chinese mulberry (M. alba L.) extracted with acidic methanol and defatted with ethyl acetate was found to exhibit five anthocyanins, including cyanidin $3-O-(6 "-O-\alpha-$ rhamnopyranosyl- $\beta$-D-glucopyranoside) (C3RG), cyanidin 3-O-(6"-O-arhamnopyranosyl- $\beta$-D-galactopyranoside) (C3RGa), cyanidin 3-O- $\beta$-D-glucopyranoside (C3G), cyanidin 3-O- $\beta$-D-galactopyranoside (C3Ga) and cyanidin 7-O- $\beta$-D-glucopyranoside (C7G) [21]. Brazil wild mulberry ( $M$. nigra L.) extracted under saponification and acid hydrolysis consisted of kuromanin (71\%) and cyanidin 3-glucosylrhamnoside (19\%) [22]. Therefore, types and quantities of detected anthocyanins/anthocyanidins depended on both internal factors (i.e., cultivars, fruit color, and stages of maturity) and external factors (i.e., climate, growing location, and extraction methods).

One of the biological functions of phenolics is that of anti-oxidative agents. The strong positive correlation between TPCs and DPPH radical scavenging activities, a moderate positive correlation with ORAC activities, and a weak positive correlation with FRAP activities suggested that most phenolics detected in Morus fruit samples are able to scavenge free radicals in the SET mechanism. On the other hand, keracyanin with strong positive correlations to antioxidant activities determined by FRAP and ORAC assays indicated that keracyanin could function as an antioxidant in both SET and HAT mechanisms. Likewise, the strong positive correlations between cyanidin and kuromanin with all three methods of antioxidant measurement suggested that these phytochemical compounds could behave as antioxidants in both SET and HAT mechanisms. The SET mechanism related to antioxidants that are able to transfer electron (electron donor) to any electron acceptors such as metals, carbonyls, and radicals. The examples of antioxidant capacity measurements under this mechanism are DPPH radical scavenging and FRAP assays [23]. In DPPH radical scavenging assay, the deep blue $\mathrm{DPPH}^{\bullet}$ radical reacts with an antioxidant to produce a yellow DPPH-H product. The FRAP assay, however, involves the ability to reduce brown ferric $\left(\mathrm{Fe}^{3+}\right)$ to indigo ferrous $\left(\mathrm{Fe}^{2+}\right)$ ions in the presence of $\mathrm{Fe}^{2+}$-stabilizing ligand such as 2,4,6-tripyridyl-s-triazine (TPTZ). The HAT mechanism is based on the ability of antioxidants to quench free radicals by hydrogen atom donation. The example of this mechanism is ORAC assay, in which antioxidant capacity is demonstrated from the kinetic curves based on competitive inhibition of chemical kinetics [23]. The peroxyl radical generated from thermogenesis of AAPH (oxidizing agents) can react with a fluorescein probe to produce non-fluorescent fluoresceinyl radicals, while antioxidants of interest are acting as competitive inhibitors, and antioxidant activity can be measured. To appropriately interpret the antioxidant actions, more than one assay is usually performed to investigate the antioxidant capacities. It was previously suggested that the antioxidant activities of anthocyanins/anthocyanidins are diverse, according to types of reactive species, environments (i.e., $\mathrm{pH}$, heat, and light exposure), and anthocyanins/anthocyanidins structures. For types of reactive species, it was found that pelargonidin is the strongest hydroxyl radical scavenger, followed by cyanidin and delphinidin, respectively [24]. However, opposite results were observed with superoxide anion scavenging capacity, in which delphinidin is the strongest scavenger, 
followed by cyanidin and pelargonidin, respectively [24]. The effect of the environment is related to sensitivity of detected anthocyanins/anthocyanidins, which also depend on extraction methods, including $\mathrm{pH}$, temperature, and light exposure [20]. Interestingly, the degree and position of hydroxyl, methoxyl, and sugar moieties of anthocyanins/anthocyanidins play a significant role in their antioxidant capacities. It was previously suggested that the increased number of free hydroxyl moieties around a pyrone ring on anthocyanin/anthocyanidin structures can elevate antioxidant capacity [25]. Besides, the presence of $3^{\prime}, 4^{\prime}$-dihydroxyl groups on the B ring (Figure 1) promotes the metal ions chelating reaction [26], while the presence of dihydroxyl moieties on the ortho-positions around the C4' position on the B ring positively affect the scavenging activity of hydroxyl radicals through iron chelation [27]. The presence of methoxyl moieties, however, reduces antioxidant capacity $[25,28]$. The addition of methoxyl moiety at the $5^{\prime}$ position on the $\mathrm{B}$ ring (petunidin) can decrease radical scavenging activity 3 -fold, compared to the one without (cyanidin) [25]. Additionally, glycosylation processes, including a number of sugar residues, types and positions of sugar, and types of glycosidic bond on anthocyanidins, vary the stability of anthocyanins, leading to different antioxidant capacities [25,29]. Interestingly, the increased number of sugar moieties at the $C 3$ position on the $C$ ring of anthocyanins (Figure 1) can decrease antioxidant activity [30]. It was previously reported that the radical scavenging activities of cyanidin were higher than kuromanin and keracyanin, respectively [25]. These results corresponded to our findings, in which cyanidin and kuromanin contents were strongly correlated to DPPH radical scavenging values, while the correlation between keracyanin and DPPH radical scavenging activity was moderated.

Not only were aqueous Morus fruit extracts involved in anti-oxidative stress, but they also exhibited strong inhibitory activities against AD key enzymes (AChE, BChE and BACE-1). The AChE inhibition ranging between $21.87 \%$ and $60.09 \%$ and the $\mathrm{BChE}$ inhibition between $21.27 \%$ and $77.02 \%$ of our aqueous Morus fruit extracts $(5 \mathrm{mg} / \mathrm{mL}$ ) are probably the results of the biological function of anthocyanins/anthocyanidins. Cyanidin was previously found to exhibit the $\mathrm{IC}_{50}$ of $14.43 \mu \mathrm{M}$ against $\mathrm{AChE}$ and a slightly higher $\mathrm{IC}_{50}$ value for $\mathrm{BChE}$ inhibition [31]. However, its glycosylated forms, keracyanin and kuromanin, exhibited lower $\mathrm{AChE}$ and $\mathrm{BChE}$ inhibition [31]. To understand the molecular mechanism of how anthocyanins/anthocyanidins inhibit cholinesterase, previous studies performed molecular docking to investigate the interactions between the enzymes and the inhibitors. Even though the molecular mechanism on AChE inhibitory interactions between the enzyme and anthocyanins/anthocyanidins is unavailable, the study on quercetin with a similar chemical structure to cyanidin (Figure 1) suggests that the inhibition occurs through blockage of the active site entrance [32]. The number of hydroxyl moieties on flavonoids seems to increase the degree of inhibition [32]. In parallel to the AChE-quercetin interactions, it is highly possible that the hydroxyl moieties at the $\mathrm{C} 3$ position in the $\mathrm{C}$ ring and at the $\mathrm{C} 5$ position in the $\mathrm{A}$ ring of cyanidin can interact with the enzyme residues in the catalytic pocket of AChE. Besides, the hydroxyl moieties at the $\mathrm{C}^{\prime}$ ' and $\mathrm{C}^{\prime}$ ' positions in the $\mathrm{B}$ ring interact with the enzyme residues in the cavity entrance of AChE. Interestingly, the glycosylation on the $\mathrm{C} 3$ hydroxyl moiety in the $\mathrm{C}$ ring decreases inhibitory activity [32]. In addition, although it is unclear whether anthocyanins/anthocyanidins exhibit BACE-1 inhibitory activity, the molecular docking study showed that cyanidin could bind to the active site of BACE-1 better than that of a well-known BACE-1 inhibitor (BACE-1 inhibitor-IV) [33]. Together, the molecular docking analysis implies that anthocyanins/anthocyanidins in aqueous Morus fruit extracts may be responsible for cholinesterase and BACE-1 inhibition.

In conclusion, among the twenty-seven mulberries used in the study, Morus sp. code SKSM 810191 exhibited high TPCs, anthocyanins and anthocyanidin contents, antioxidant activities, and AD key enzyme inhibitions, highlighting its potential for phytochemical compounds of a rich mulberry resource with health benefits against $\mathrm{AD}$ occurrence. 


\section{Materials and Methods}

\subsection{Mulberry Collection, Preparation, and Extraction}

The fruits of uniform color and ripening stage of twenty-seven Morus spp. were collected from the Queen Sirikit Department of Sericulture Center (Kanchanaburi), Thailand. The cultivars, sample code, and collector are listed in Supplementary Table S1. Mulberry fruits were cleaned once with tap water and twice with deionized water (DI). After that, the samples were freeze-dried (Heto PowerDry PL9000, Heto Lab Equipment, Allerød, Denmark) for 3 days. Dry samples were ground into fine powder using a grinder (Philips 600W series, Philips Electronics Co., Ltd., Jakarta, Indonesia), and packed in vacuum aluminum foil bags before extraction. The aqueous extract was then prepared, as previously described [12].

The colors of both fresh and dry samples were measured using a spectrophotometer (ColorFlex EZ, Hunter Associates Laboratory, Virginia, USA) and expressed as Hunter-Lab units, including L representing dark (0) to white (100) colors, a representing green $(-)$ to red $(+)$ colors and b representing blue $(-)$ to yellow $(+)$ colors. The moisture contents of powdery samples were analyzed using a Halogen moisture analyzer (HE53 series, Mettler-Toledo AG, Greifensee, Switzerland). The data of colors and moisture contents are showed in Supplementary Table S2.

\subsection{Evaluation of Antioxidant Activity}

The antioxidant activities including 2,2-diphenyl-1-picrylhydrazyl (DPPH) radical scavenging activity, oxygen radical absorbance capacity (ORAC), and ferric ion reducing antioxidant power (FRAP) assays of Morus fruit extracts, were performed using the well-established protocols indicated in Thuphairo et al. 2019 [34-37].

\subsection{Determination of Total Phenolic Contents, Anthocyanin and Anthocyanidin}

Total phenolic contents (TPCs) of Morus fruit extracts were determined using Folin-Ciocalteu reagent, as described by Thuphairo et al., 2019 [38]. Gallic acid (10-200 $\mu \mathrm{g} / \mathrm{mL})$ was used as a standard, and the TPCs were reported as mg gallic acid equivalent (GAE)/g dried matter (DW) [38].

To determine anthocyanidins, Morus fruit powder was extracted using acid hydrolysis by dispersion of the powdered sample $(500 \mathrm{mg})$ in $50 \%(v / v)$ aqueous methanol containing $2 \mathrm{~N} \mathrm{HCl}$ $\left(5 \mathrm{~mL}\right.$ ). The extract was incubated in a $100 \pm 2{ }^{\circ} \mathrm{C}$ water bath (TW20 series from Julabo GmbH, Seelbach, Germany) for $1 \mathrm{~h}$ and filtered through a $0.22 \mu \mathrm{M}$ PTFE membrane syringe filter into a $2 \mathrm{~mL}$ HPLC vial. The identification of anthocyanidins of Morus fruit extracts $(20 \mu \mathrm{L})$ was achieved by an UtiMate 3000 HPLC system with diode array and multiple-wavelength detectors from Thermo Fisher Scientific (Dreieich, Germany) utilizing a $5 \mu \mathrm{m}$ ReproSil-Pur ${ }^{\circledR}$ ODS-3 column $(250 \times 4.6 \mathrm{~mm})$ from Dr. Maisch GmbH (Ammerbuch, Germany). Milli-Q water (18.2 M .cm conductivity) containing $0.4 \%(v / v)$ TFA (solvent A) and acetonitrile containing $0.4 \% v / v$ TFA (solvent B) were used as isocratic mobile phase at $82 \%$ solvent $\mathrm{A}$ and $18 \%$ solvent $\mathrm{B}$ with a constant flow rate of $1.0 \mathrm{~mL} / \mathrm{min}$ over $60 \mathrm{~min}$. The anthocyanidins was visualized at $530 \mathrm{~nm}$ using a Chromeleon ${ }^{\mathrm{TM}}$ Chromatography Data System (CDS) software (Thermo Fisher Scientific, Dreieich, Germany). The retention times $\left(R_{t}\right)$ and UV-Vis spectral fingerprints of standards including cyanidin ( $\geq 96.0 \%$ HPLC), delphinidin ( $\geq 97.0 \%$ HPLC), pelargonidin ( $\geq 97.0 \%$ HPLC), peonidin ( $\geq 97.0 \%$ HPLC), and petunidin ( $\geq 95.0 \%$ HPLC) from Extrasynthese (Genay, France) were used to confirm the existence of the anthocyanidins in Morus fruit extracts.

For anthocyanin analysis, Morus fruit extracts were prepared similarly to those for anthocynidins. However, low concentration of acid $(2 \%(v / v) \mathrm{HCl}$ in $5 \mathrm{~mL}$ of $50 \%(v / v)$ aqueous methanol) was applied to the extraction to stabilize anthocyanins. The HPLC analysis was performed utilizing a constant flow rate of $1 \mathrm{~mL} / \mathrm{min}$ at ambient temperature. The solvent system was shown in Table 5 as previously described [12]. 
Table 5. Solvent system of anthocyanin analysis using HPLC analysis.

\begin{tabular}{ccc}
\hline Time (min) & Solvent A & Solvent B \\
\hline 0 & 88 & 12 \\
6 & 88 & 12 \\
8 & 85 & 15 \\
25 & 85 & 15 \\
25 & 88 & 12 \\
30 & 88 & 12
\end{tabular}

Solvent A = Milli-Q water containing $0.4 \%(v / v)$ TFA; solvent $\mathrm{B}=$ acetonitrile containing $0.4 \%(v / v)$ TFA.

The existence of the anthocyanins was visualized at $525 \mathrm{~nm}$ and compared $R_{t}$ and UV-Vis spectral fingerprints with standards including callistephin (pelargonidin 3-O-glucoside) ( $\geq 95.0 \%$ HPLC), cyanidin 3-O-sophoroside ( $\geq 95.0 \%$ HPLC), cyanin (cyanidin 3,5-di-O-glucoside) $(\geq 97.0 \%$ HPLC), delphin (delphinidin 3,5-di-O-glucoside) $(\geq 97.0 \%$ HPLC), ideain (cyanidin 3-O-galactoside) $(\geq 97.0 \%$ HPLC), keracyanin (cyanidin 3-O-rutinoside) ( $\geq 96.0 \%$ HPLC), kuromanin (cyanidin 3-O-glucoside) ( $\geq 96.0 \%$ HPLC), malvidin (malvidin 3-O-beta-D-glucoside) $(\geq 97.0 \%$ HPLC), and pelargonidin 3-O-rutinoside ( $\geq 90.0 \%$ HPLC) from Extrasynthese (Genay, France).

\subsection{Determination of Cholinesterases and Beta-secretase 1 (BACE-1) Inhibitory Activities}

Cholinesterases (AChE and $\mathrm{BChE}$ ) inhibitory activities of Morus fruit extracts were performed as previously reported [38-40]. Briefly, the enzyme assay consisting of $20 \mathrm{ng}$ of Electrophorus electricus AChE (1000 units/mg, $100 \mu \mathrm{L}$ ) in $50 \mathrm{mM} \mathrm{KPB} \mathrm{(pH} \mathrm{7.0),} 16 \mathrm{mM}$ 5,5-dithio-bis-(2-nitrobenzoic acid) (DTNB, $10 \mu \mathrm{L}), 0.8 \mathrm{mM}$ acetylthiocholine $(40 \mu \mathrm{L})$ in $50 \mathrm{mM} \mathrm{KPB}(\mathrm{pH} 7.0)$, and the extract $(50 \mu \mathrm{L})$ was detected at $412 \mathrm{~nm}$ using a microplate reader (Synergy ${ }^{\mathrm{TM}}$ HT 96-well UV-Vis spectrophotometer with a Gen5 data analysis software from BioTek Instruments, Inc., Winooski, VT, USA). The percentage of inhibition was then calculated as follows:

$$
\% \text { inhibition }=\left(1-\frac{B-b}{A-a}\right) \times 100
$$

where $A$ is the initial velocity of the reaction with enzyme, $a$ is the initial velocity of the reaction without enzyme, $B$ is the initial velocity of the enzyme reaction with extract, and $b$ is the initial velocity of the reaction with extract but without enzyme.

The BChE inhibitory activities of Morus fruit extracts were determined similarly to AChE, except that $100 \mathrm{ng}$ equine serum $\mathrm{BChE}(\geq 10$ units/mg protein, $100 \mu \mathrm{L})$ in $50 \mathrm{mM} \mathrm{KPB} \mathrm{(pH} \mathrm{7.0)} \mathrm{containing}$ $1 \mathrm{mM} \mathrm{MgCl}$ and $0.1 \mathrm{mM}$ butyrylthiocholine (BTCh) in $50 \mathrm{mM} \mathrm{KPB} \mathrm{(pH} \mathrm{7.0)} \mathrm{were} \mathrm{used} \mathrm{as} \mathrm{the} \mathrm{reaction}$ enzyme and substrate, respectively $[38,39]$. All enzymes, chemicals and reagents for cholinesterase inhibitions were purchased from Sigma-Aldrich (St. Louis, MO, USA).

The BACE-1 activity was determined utilizing the fluorescence resonance energy transfer (FRET) method on a BACE-1 activity detection kit (Sigma-Aldrich). The manufacturer's instructions were followed, and the results were expressed as a percentage of BACE-1 inhibition as above.

\subsection{Statistical Analysis}

All experiments were carried out in triplicate $(n=3)$ and expressed as mean \pm standard deviation (SD). One-way Analysis of Variance (ANOVA) and Duncan's multiple comparison tests were performed to determine the significant differences between values with $p<0.05$. Two-way Pearson bivariated correlation test was performed to determine the significantly different correlation between values with $p \leq 0.01$ and $p \leq 0.05$. Mean value of all variables investigated in the samples were also subjected to principal component analysis (PCA) and agglomerative hierarchical clustering analysis (AHC) using the XLSTAT-base version 2019.3.2 software (Addinsoft, New York, NY, USA). 
Supplementary Materials: Supplementary Table S1: Images of twenty-seven cultivars, sample codes, and collectors of Morus fruit samples used in this experiment. Supplementary Table S2: Color (where L describes darkness $(-)$ to lightness $(+)$, a describes green $(-)$ to red $(+)$ colors, and b describes indigo $(-)$ to yellow $(+)$ colors) and the percentage $(\%)$ of moisture content of fresh and freeze-dried Morus fruit samples. Supplementary Figure S1: High-performance liquid chromatography (HPLC) chromatograms of (A.) cyanidin chloride standard, and anthocyanidin analyses of Morus fruit extracts including (B.) Morus 'Krua', (C.) Morus 'Jak', (D.) Morus 'Pai', (E.) Morus 'Pai-Ubon', (F.) Morus 'Poe', (G.) Morus 'Mae Luke On', (H.) Morus 'Som', (I.) Morus 'Som Yai', (J.) Morus 'Sida', (K.) Morus 'Kun Pai', (L.) Morus 'Nakhon Ratchasima 60', (M.) Morus 'Buri Ram 51', (N.) Morus 'Buri Ram 60', (O.) Morus 'Si Sa Ket 33', (P.) Morus 'Number 44', (Q.) Morus sp. code SKSM 820281, (R.) Morus sp. code SKSM 14-13-20, (S.) Morus sp. code SKSM 040691, (T.) Morus sp. code SKSM 810191', (U.) Morus sp. code SKSM 810391, (V.) Morus sp. code SRCM 9124-12, (W.) Morus sp. code SRCM 9801-465, (X.) Morus sp. code SRCM 9801-535, (Y.) Morus sp. code SRCM 9801-833, (Z.) Morus sp. code SRCM 9806-271, (AA.) Morus sp. code SRCM 9806-287, and (AB.) Morus sp. code SRCM 9809-34. The retention times $\left(R_{t}\right)$ of cyanidin chloride in Morus fruit extracts were also indicated. Supplementary Figure S2: High-performance liquid chromatography (HPLC) chromatograms of (A.) kuromanin and keracyanin standards, and anthocyanin analyses of Morus fruit extracts including (B.) Morus 'Krua', (C.) Morus 'Jak', (D.) Morus 'Pai', (E.) Morus 'Pai-Ubon', (F.) Morus 'Poe', (G.) Morus 'Mae Luke On', (H.) Morus 'Som', (I.) Morus ‘Som Yai', (J.) Morus 'Sida', (K.) Morus 'Kun Pai', (L.) Morus 'Nakhon Ratchasima 60', (M.) Morus ‘Buri Ram 51', (N.) Morus 'Buri Ram 60', (O.) Morus 'Si Sa Ket 33', (P.) Morus 'Number 44', (Q.) Morus sp. code SKSM 820281, (R.) Morus sp. code SKSM 14-13-20, (S.) Morus sp. code SKSM 040691, (T.) Morus sp. code SKSM 810191', (U.) Morus sp. code SKSM 810391, (V.) Morus sp. code SRCM 9124-12, (W.) Morus sp. code SRCM 9801-465, (X.) Morus sp. code SRCM 9801-535, (Y.) Morus sp. code SRCM 9801-833, (Z.) Morus sp. code SRCM 9806-271, (AA.) Morus sp. code SRCM 9806-287, and (AB.) Morus sp. code SRCM 9809-34. The retention times $\left(R_{t}\right)$ of kuromanin and keracyanin in Morus fruit extracts were also indicated.

Author Contributions: P.T. designed the research, interpreted the results, generated the figures and tables, wrote the manuscript, reviewed the manuscript. V.S. performed experiments and statistically analysis. K.N.J. performed statistically analysis and wrote the manuscript (PCA and AHC). B.J., S.T. and K.P. initiated technical support and provided samples. S.T. performed experiments. W.I. performed experiments, prepared manuscript. D.S. and S.C. suggested and reviewed the manuscript. U.S. designed the research, supervised, performed experiments, interpreted the results, generated the figures and tables, wrote the manuscript, reviewed the manuscript. All authors have read and agree to the published version of the manuscript.

Funding: This study was supported by Biodiversity-Based Economy Development Office (BEDO) (grant No. 22/2561), and partially supported by Grants-in-Aid from JSPS Core to-Core Program B. Asia-Africa Science Platforms

Conflicts of Interest: All authors declare that there are no conflicts of interest.

\section{References}

1. Lopez-Otin, C.; Blasco, M.A.; Partridge, L.; Serrano, M.; Kroemer, G. The hallmarks of aging. Cell 2013, 153, 1194-1217. [CrossRef] [PubMed]

2. Emmerzaal, T.L.; Kiliaan, A.J.; Gustafson, D.R. 2003-2013: A decade of body mass index, Alzheimer's disease, and dementia. J. Alzheimers Dis. 2015, 43, 739-755. [CrossRef] [PubMed]

3. Casey, D.A.; Antimisiaris, D.; O'Brien, J. Drugs for Alzheimer's disease: Are they effective? Pharm. Therap. 2010, 35, 208-211.

4. Hosen, S.M.Z.; Rubayed, M.; Dash, R.; Junaid, M.; Mitra, S.; Alam, M.S.; Dey, R. Prospecting and structural insight into the binding of novel plant-derived molecules of Leea indica as inhibitors of BACE1. Curr. Pharm. Des. 2018, 24, 3972-3979. [CrossRef]

5. Youn, K.; Lee, J.; Ho, C.T.; Jun, M. Discovery of polymethoxyflavones from black ginger (Kaempferia parviflora) as potential beta-secretase (BACE1) inhibitors. J. Funct. Foods 2016, 20, 567-574. [CrossRef]

6. Seong, S.H.; Ha, M.T.; Min, B.S.; Jung, H.A.; Choi, J.S. Moracin derivatives from Morus Radix as dual BACE1 and cholinesterase inhibitors with antioxidant and anti-glycation capacities. Life Sci. 2018, 210, 20-28. [CrossRef]

7. Ercisli, S.; Orhan, E. Chemical composition of white (Morus alba), red (Morus rubra) and black (Morus nigra) mulberry fruits. Food Chem. 2007, 103, 1380-1384. [CrossRef]

8. Yuan, Q.X.; Zhao, L.Y. The Mulberry (Morus alba L.) Fruit-A review of characteristic components and health benefits. J. Agr. Food Chem. 2017, 65, 10383-10394. [CrossRef]

9. Tsuda, T. Anthocyanins as functional food factors-Chemistry, nutrition and health promotion. Food Sci. Technol. Res. 2012, 18, 315-324. [CrossRef]

10. Kong, J.M.; Chia, L.S.; Goh, N.K.; Chia, T.F.; Brouillard, R. Analysis and biological activities of anthocyanins. Phytochemistry 2003, 64, 923-933. [CrossRef] 
11. Hidalgo, G.I.; Almajano, M.P. Red fruits: Extraction of antioxidants, phenolic content, and radical scavenging determination: A Review. Antioxidants (Basel) 2017, 6, 7. [CrossRef] [PubMed]

12. Suttisansanee, U.; Charoenkiatkul, S.; Jongruaysup, B.; Tabtimsri, S.; Siriwan, D.; Temviriyanukul, P. Mulberry fruit cultivar 'Chiang Mai' prevents beta-amyloid toxicity in PC12 neuronal cells and in a Drosophila model of Alzheimer's disease. Molecules 2020, 25, 1837. [CrossRef] [PubMed]

13. Özgen, M.; Serçe, S.; Kaya, C. Phytochemical and antioxidant properties of anthocyanin-rich Morus nigra and Morus rubra fruits. Sci. Hortic. 2009, 119, 275-279.

14. Lin, J.-Y.; Tang, C.-Y. Determination of total phenolic and flavonoid contents in selected fruits and vegetables, as well as their stimulatory effects on mouse splenocyte proliferation. Food Chem. 2007, 101, 140-147. [CrossRef]

15. Isabelle, M.; Lee, B.L.; Ong, C.N.; Liu, X.; Huang, D. Peroxyl radical scavenging capacity, polyphenolics, and lipophilic antioxidant profiles of mulberry fruits cultivated in southern China. J. Agric. Food Chem. 2008, 56, 9410-9416. [CrossRef]

16. Bae, S.-H.; Suh, H.-J. Antioxidant activities of five different mulberry cultivars in Korea. LWT Food Sci. Technol. 2007, 40, 955-962. [CrossRef]

17. Natic, M.M.; Dabic, D.C.; Papetti, A.; Fotiric Aksic, M.M.; Ognjanov, V.; Ljubojevic, M.; Tesic, Z. Analysis and characterisation of phytochemicals in mulberry (Morus alba L.) fruits grown in Vojvodina, North Serbia. Food Chem. 2015, 171, 128-136. [CrossRef] [PubMed]

18. Castañeda-Ovando, A.; Pacheco-Hernández, M.D.L.; Páez-Hernández, M.E.; Rodríguez, J.A.; Galán-Vidal, C.A. Chemical studies of anthocyanins: A review. Food Chem. 2009, 113, 859-871.

19. Seeram, N.P.; Momin, R.A.; Nair, M.G.; Bourquin, L.D. Cyclooxygenase inhibitory and antioxidant cyanidin glycosides in cherries and berries. Phytomedicine 2001, 8, 362-369. [CrossRef]

20. Qin, C.; Li, Y.; Niu, W.; Ding, Y.; Zhang, R.; Shang, X. Analysis and characterisation of anthocyanins in mulberry fruit. Czech J. Food Sci. 2010, 28, 17-126. [CrossRef]

21. Du, Q.; Zheng, J.; Xu, Y. Composition of anthocyanins in mulberry and their antioxidant activity. J. Food Compos. Anal. 2008, 21, 390-395. [CrossRef]

22. Hassimotto, N.M.A.; Genovese, M.I.; Lajolo, F.M. Identification and characterisation of anthocyanins from wild mulberry (Morus Nigra L.) growing in Brazil. Food Sci. Technol. Int. 2007, 13, 17-25. [CrossRef]

23. Miguel, M.G. Anthocyanins: Antioxidant and/or anti-inflammatory activities. J. Appl. Pharm. Sci. 2011, 1, 7-15.

24. Tsuda, T.; Shiga, K.; Ohshima, K.; Kawakishi, S.; Osawa, T. Inhibition of lipid peroxidation and the active oxygen radical scavenging effect of anthocyanin pigments isolated from Phaseolus vulgaris L. Biochem. Pharm. 1996, 52, 1033-1039. [CrossRef]

25. Kähkönen, M.P.; Heinonen, M. Antioxidant activity of anthocyanins and their aglycons. J. Agr. Food Chem. 2003, 51, 628-633.

26. Sarma, A.D.; Sreelakshmi, Y.; Sharma, R. Antioxidant ability of anthocyanins against ascorbic acid oxidation. Phytochemistry 1997, 45, 671-674. [CrossRef]

27. Noda, Y.; Kneyuki, T.; Igarashi, K.; Mori, A.; Packer, L. Antioxidant activity of nasunin, an anthocyanin in eggplant peels. Toxicology 2000, 148, 119-123. [CrossRef]

28. Muselík, J.; García-Alonso, M.; Martín-López, M.P.; Žemlička, M.; Rivas-Gonzalo, J.C. Measurement of antioxidant activity of wine catechins, procyanidins, anthocyanins and pyranoanthocyanins. Int. J. Mol. Sci. 2007, 8, 797-809.

29. Wang, Z.; Lin, Y.; Li, T.; Dai, F.; Luo, G.; Xiao, G.; Tang, C. Phenolic profiles and antioxidant capacities of mulberry (Morus atropurpurea Roxb.) juices from different cultivars. Int. J. Food Prop. 2019, 22, 1340-1352. [CrossRef]

30. Estévez, L.; Mosquera, R.A. Molecular structure and antioxidant properties of delphinidin. J. Phys. Chem. A 2008, 112, 10614-10623.

31. Szwajgier, D. Anticholinesterase activities of selected polyphenols-A short report. Pol. J. Food Nutr. Sci. 2014, 64, 59-64. [CrossRef]

32. Khan, M.T.; Orhan, I.; Senol, F.S.; Kartal, M.; Sener, B.; Dvorska, M.; Smejkal, K.; Slapetova, T. Cholinesterase inhibitory activities of some flavonoid derivatives and chosen xanthone and their molecular docking studies. Chem. Biol. Interact. 2009, 181, 383-389. [CrossRef] 
33. Dhananjayan, K.; Arunachalam, S.; Anand Raj, B. Targeting BACE1 (beta-secretase) through polyphenolic compounds-A computational in silico approach with emphasis on binding site analysis. J. Comput. Methods Mol. Des. 2014, 4, 14-24.

34. Benzie, I.F.; Strain, J.J. The ferric reducing ability of plasma (FRAP) as a measure of "antioxidant power": The FRAP assay. Anal. Biochem. 1996, 239, 70-76. [CrossRef] [PubMed]

35. Fukumoto, L.R.; Mazza, G. Assessing antioxidant and prooxidant activities of phenolic compounds. J. Agric. Food Chem. 2000, 48, 3597-3604. [CrossRef] [PubMed]

36. Ou, B.; Hampsch-Woodill, M.; Prior, R.L. Development and validation of an improved oxygen radical absorbance capacity assay using fluorescein as the fluorescent probe. J. Agric. Food Chem. 2001, 49, 4619-4626. [CrossRef] [PubMed]

37. Sripum, C.; Kukreja, R.K.; Charoenkiatkul, S.; Kriengsinyos, W.; Suttisansanee, U. The effect of extraction conditions on antioxidant activities and total phenolic contents of different processed Thai Jasmine rice. Int. Food Res. J. 2017, 24, 1644-1650.

38. Thuphairo, K.; Sornchan, P.; Suttisansanee, U. Bioactive compounds, antioxidant activity and inhibition of key enzymes relevant to Alzheimer's disease from sweet Pepper (Capsicum annuum) extracts. Prev. Nutr. Food Sci. 2019, 24, 327-337. [CrossRef]

39. Jung, H.A.; Min, B.S.; Yokozawa, T.; Lee, J.H.; Kim, Y.S.; Choi, J.S. Anti-Alzheimer and antioxidant activities of Coptidis Rhizoma alkaloids. Biol. Pharm. Bull. 2009, 32, 1433-1438. [CrossRef]

40. Nantakornsuttanan, N.; Thuphairo, K.; Kukreja, R.K.; Charoenkiatkul, S.; Suttisansanee, U. Anti-cholinesterase inhibitory activities of different varieties of chili peppers extracts. Int. Food Res. J. 2016, 23, 1953-1959.

Sample Availability: Samples of the compounds are not available from the authors.

(C) 2020 by the authors. Licensee MDPI, Basel, Switzerland. This article is an open access article distributed under the terms and conditions of the Creative Commons Attribution (CC BY) license (http://creativecommons.org/licenses/by/4.0/). 